\title{
HST PanCET program: Non-detection of atmospheric escape in the warm Saturn-sized planet WASP-29 b
}

\author{
L. A. dos Santos ${ }^{1}$, V. Bourrier ${ }^{1}$, D. Ehrenreich ${ }^{1}$, J. Sanz-Forcada ${ }^{2}$, M. López-Morales ${ }^{3}$, D. K. Sing ${ }^{4,5}$, \\ A. García Muñoz ${ }^{6}$, G. W. Henry ${ }^{7}$, P. Lavvas ${ }^{8}$, A. Lecavelier des Etangs ${ }^{9}$, T. Mikal-Evans ${ }^{10}$, A. Vidal-Madjar ${ }^{9}$, and \\ H. R. Wakeford ${ }^{11}$
}

1 Observatoire astronomique de l'Université de Genève, Chemin Pegasi 51b, 1290 Versoix, Switzerland e-mail: Leonardo.dosSantos@unige.ch

2 Centro de Astrobiología (CSIC-INTA), E-28692 Villanueva de la Cañada, Madrid, Spain

3 Center for Astrophysics | Harvard \& Smithsonian, 60 Garden Street, Cambridge, MA 02138, USA

4 Department of Earth \& Planetary Sciences, Johns Hopkins University, Baltimore, MD, USA

5 Department of Physics \& Astronomy, Johns Hopkins University, Baltimore, MD, USA

6 AIM, CEA, CNRS, Université Paris-Saclay, Université de Paris, F-91191 Gif-sur-Yvette, France

7 Tennessee State University, Center of Excellence in Information Systems, Nashville, TN 37203, USA

${ }^{8}$ Groupe de Spectrométrie Moléculaire et Atmosphérique, Université de Reims, Champagne-Ardenne, CNRS UMR F-7331, France

${ }^{9}$ Institut d'Astrophysique de Paris, CNRS, UMR 7095 \& Sorbonne Universités UPMC Paris 6, 98 bis bd Arago, 75014 Paris, France

${ }^{10}$ Kavli Institute for Astrophysics and Space Research, Massachusetts Institute of Technology, Cambridge, MA, USA

11 School of Physics, University of Bristol, HH Wills Physics Laboratory, Tyndall Avenue, Bristol BS8 1TL, UK

Received 03 February 2021; accepted 29 March 2021

\begin{abstract}
Short-period gas giant exoplanets are susceptible to intense atmospheric escape due to their large scale heights and strong high-energy irradiation. This process is thought to occur ubiquitously, but to date we have only detected direct evidence of atmospheric escape in hot Jupiters and warm Neptunes. The latter planets are particularly more sensitive to escape-driven evolution as a result of their lower gravities with respect to Jupiter-sized planets. But the paucity of cases for intermediate, Saturn-sized exoplanets at varying levels of irradiation precludes a detailed understanding of the underlying physics in atmospheric escape of hot gas giants. Aiming to address this issue, our objectives here are to assess the high-energy environment of the warm $\left(T_{\mathrm{eq}}=970 \mathrm{~K}\right)$ Saturn WASP-29 b and search for signatures of atmospheric escape. We used far-ultraviolet (FUV) observations from the Hubble Space Telescope to analyze the flux time series of $\mathrm{H}_{\text {I }} \mathrm{C}_{\text {II }}, \mathrm{Si}$ III, Si IV, and N v during the transit of WASP-29 b. At 88 pc, a large portion of the Lyman- $\alpha$ core of the $\mathrm{K} 4 \mathrm{~V}$-type host WASP-29 is attenuated by interstellar medium absorption, limiting our ability to probe the escape of $\mathrm{H}$ at velocities between $[-84,+35] \mathrm{km} \mathrm{s}^{-1}$. At $3 \sigma$ confidence, we rule out any in-transit absorption of $\mathrm{H}_{\mathrm{I}}$ larger than $92 \%$ in the Lyman- $\alpha$ blue wing and $19 \%$ in the red wing. We found an in-transit flux decrease of $39 \%_{-11 \%}^{+12 \%}$ in the ground-state $\mathrm{C}_{\text {II }}$ emission line at $1334.5 \AA$. But due to this signal being significantly present in only one visit, it is difficult to attribute a planetary or stellar origin to the groundstate $\mathrm{C}_{\text {II }}$ signal. We place $3 \sigma$ absorption upper limits of $40 \%, 49 \%$, and $24 \%$ on Si III, Si IV, and for excited-state $\mathrm{C}_{\text {II }}$ at $1335.7 \AA$, respectively. Low activity levels and the faint X-ray luminosity suggest that WASP-29 is an old, inactive star. Nonetheless, an energylimited approximation combined with the reconstructed EUV spectrum of the host suggests that the planet is losing its atmosphere at a relatively large rate of $4 \times 10^{9} \mathrm{~g} \mathrm{~s}^{-1}$. The non-detection at Lyman- $\alpha$ could be partly explained by a low fraction of escaping neutral hydrogen, or by the state of fast radiative blow-out we infer from the reconstructed Lyman- $\alpha$ line.
\end{abstract}

Key words. Stars: individual: WASP-29 - stars: chromospheres - planets and satellites: atmospheres - ISM: kinematics and dynamics

\section{Introduction}

The large population of transiting exoplanets yields the best opportunity to perform demographic studies aiming to characterize and understand their atmospheres. One of the main techniques to do so is transmission spectroscopy, which consists in measuring wavelength-dependent changes in the stellar spectrum when a planet transits its host star. The largest atmospheric signals of exoplanets detected to date have been obtained with transmission spectroscopy in far- and near-ultraviolet (FUV, NUV). This wavelength range traces the outermost parts of the atmospheres of transiting planets, where the lightest particles are susceptible to atmospheric escape. This has been observed in planets with
$\mathrm{H}$ - and He-dominated atmospheres, such as the warm Neptunes GJ 436 b (Kulow et al. 2014, Ehrenreich et al. 2015, Bourrier et al. 2016; Lavie et al. 2017; dos Santos et al. 2019), GJ 3470 b (Bourrier et al.|2018b), the hot-Jupiters HD 209458 b (VidalMadjar et al. 2003, 2004; Ehrenreich et al. 2008; Vidal-Madjar et al. 2013), HD 189733 b (Lecavelier des Etangs et al.|2010. 2012, Bourrier et al. 2013), WASP-12 b (Fossati et al. 2010) and WASP-121 b (Sing et al.2019), and tentatively for the temperate sub-Neptune K2-18 b (dos Santos et al.|2020b).

These signals point to the presence of $\mathrm{H}$-rich, extended atmospheres and mass loss in both ends of the gas giant demographic, but the middle range that includes hot and warm Saturnsized planets remains poorly explored (Fig. 11). Direct and indi- 
rect observational evidence has shown that mass loss is one of the most important drivers for atmospheric evolution in shortperiod Neptune-sized exoplanets, as well as planetary migration and formation (e.g., Szabó \& Kiss 2011; Beaugé \& Nesvorný 2013; Mazeh et al. 2016; Helled et al. 2016; Matsakos \& Königl 2016; Owen \& Lai 2018; Bourrier et al. 2018b; McDonald et al. 2019; Armstrong et al. 2020); although, readers can refer to $\mathrm{Cu}$ billos et al. (2017), Kite et al. (2019), and Vidotto \& Cleary (2020) for possible counterpoints. Such a conclusion is backed up by a strong theoretical framework as well (e.g., Lecavelier Des Etangs 2007, Rogers et al. 2011, Sanz-Forcada et al. 2011; Kurokawa \& Nakamoto 2014; Tian 2015; S Salz et al. |2016a). For a detailed review on the subject, readers can look to Owen (2019). This atmospheric evolution results in close-in Neptunes having short lifetimes, imprinting a feature in the population of known transiting exoplanets known as the hot-Neptune desert (Szabó \& Kiss 2011; Mazeh et al. 2016, Bourrier et al.|2018b).

Hot Jupiters have been shown to be too massive to lose a significant fraction of their atmospheres due to photoevaporation (e.g., García Muñoz 2007; Hubbard et al. 2007; Ehrenreich \& Désert 2011; Allan \& Vidotto 2019). However, García Muñoz \& Schneider (2019) have proposed that ultra-hot Jupiters orbiting early-type stars, such as KELT-9 b, may have lifetimes of less than 1 Gyr due to near-ultraviolet energy deposited through Balmer absorption. The lack of direct observational constraints on mass loss for Saturn-sized planets at the borders of the hotNeptune desert contributes to the aforementioned poorly explored demographic. By directly detecting and assessing the current atmospheric rate and other properties of a sample of planetary systems, it is possible to tease apart the respective roles of planetary formation, migration, stellar wind, and photoevaporation in shaping the observed demographics of gas giants (Jin) et al. 2014; Owen \& Lai 2018; Vidotto \& Cleary 2020).

One of the objectives of the Hubble Space Telescope Panchromatic Comparative Exoplanetology (HST PanCET) program is to address questions about atmospheres of gas giant transiting planets at a demographic level. One of the targets of this program is WASP-29 b, a low-density and moderately irradiated Saturn-sized planet orbiting every $3.92 \mathrm{~d}$ around a K4V-type star first discovered by Hellier et al. (2010, the stellar and planetary parameters are listed in Table 1). A previous attempt at studying the atmosphere of WASP-29 b using ground-based, lowresolution $(R<1000)$ transmission spectroscopy between 515 and $720 \mathrm{~nm}$ yielded a featureless spectrum (Gibson et al. 2013). In particular, the lack of a broad $\mathrm{Na}$ feature indicates that either the planet does not have neutral $\mathrm{Na}$ in its upper atmosphere, or that clouds and hazes obscure the broad wings of the feature, resulting in a narrow absorption that is not detectable at low spectral resolution.

In this paper, we report on the data analysis and results of several transits of WASP-29 b observed with HST in FUV, aiming to detect and study its upper atmosphere. In particular, we are interested in addressing the following open issues: i) how the high-energy environment around WASP-29 b influences the observable signatures of atmospheric escape; ii) what escape rates should we expect for hot Saturns similar to WASP-29 b; and iii) whether it shows signals of hydrodynamic escape such as hot Jupiters and warm Neptunes. This manuscript has the following structure: in Sect. 2 we describe the HST observations and data reduction; in Sections 3 and 4 we present the analysis of the flux time series and the search for planetary signals in transmission; in Sect. 5] we discuss the reconstruction of the unobservable high-energy spectrum of WASP-29; and in Sect.6 we present the conclusions of this work.
Table 1. Stellar and planetary parameters of the WASP-29 system.

\begin{tabular}{lcc}
\hline \hline \multicolumn{1}{c}{ Stellar parameters of WASP-29 } & Ref. \\
\hline Radius & $0.813_{-0.015}^{+0.033} \mathrm{R}_{\odot}$ & (a) \\
Mass & $0.825 \pm 0.033 \mathrm{M}_{\odot}$ & (b) \\
Eff. temperature & $4731_{-95}^{+44} \mathrm{~K}$ & (a) \\
Proj. rot. velocity & $1.5 \pm 0.6 \mathrm{~km} \mathrm{~s}^{-1}$ & (b) \\
Radial velocity & $24.48 \pm 0.43 \mathrm{~km} \mathrm{~s}^{-1}$ & (a) \\
Distance & $87.82 \pm 0.31 \mathrm{pc}$ & (a) \\
Sp. type & $\mathrm{K} 4 \mathrm{~V}$ & (c) \\
\hline \multicolumn{1}{c}{ Planetary parameters of WASP-29 } & Ref. \\
\hline Radius & $0.792_{-0.035}^{+0.056} \mathrm{R}_{\mathrm{Jup}}$ & (b) \\
Mass & $0.245_{-0.022}^{+0.023} \mathrm{M}_{\mathrm{Jup}}$ & (b) \\
Density & $0.61 \pm 0.12 \mathrm{~g}^{-3}$ & (b) \\
Eq. temperature & $970.0_{-31.0}^{+32.0} \mathrm{~K}$ & (c) \\
Orbital period & $3.9227186 \pm 0.0000068 \mathrm{~d}$ & (b) \\
Semi-major axis & $0.04566_{-0.00006}^{+0.0006} \mathrm{au}$ & (b) \\
Ref. time (BJD) & $2455830.18886 \pm 0.00016$ & (b) \\
Transit duration & $2.6486_{-0.0151}^{+0.0170} \mathrm{~h}$ & (b) \\
Orbital inclination & $88.80 \pm 0.70 \mathrm{deg}$ & (b) \\
Eccentricity & $<0.059$ & (b) \\
\hline
\end{tabular}

References. (a) Gaia Collaboration et al. (2018), (b) Bonomo et al. (2017), (c) Gibson et al. (2013).

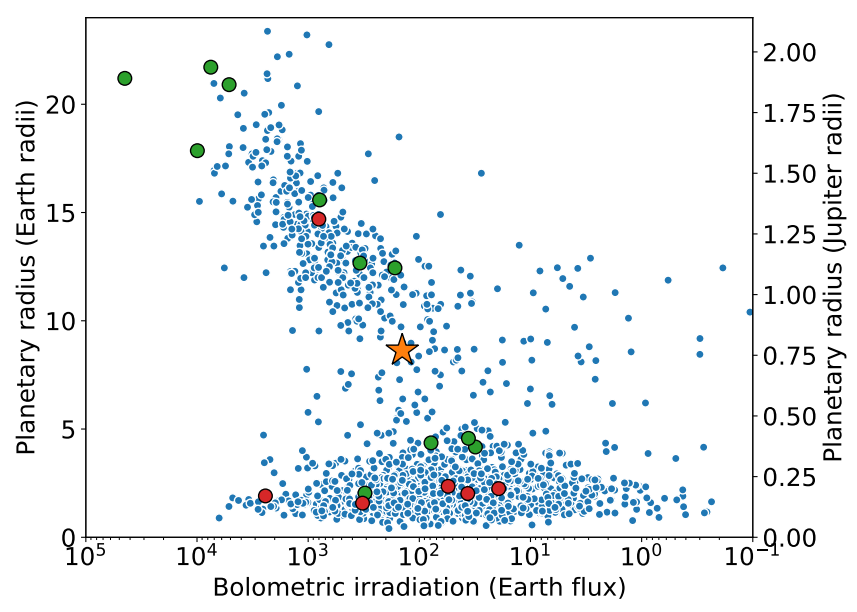

Fig. 1. Population of exoplanets with known radius and bolometric irradiation (taken from the NASA Exoplanets Archive on 01 Feb 2021). Planets with confirmed atmospheric escape signatures of $\mathrm{H}$, He or metals are shown as green symbols. Non-detections are shown as red symbols. WASP-29 b is represented as a star symbol.

\section{Description of observations}

We observed six transits of WASP-29 b in the HST PanCET program (GO-14767, PIs: D. Sing and M. López-Morales), of which three were performed with the Cosmic Origins Spectrograph (COS; Visits A, B, and C) and three with the Space Telescope Imaging Spectrograph (STIS; Visits D, E, and F). Each transit was observed in a single visit with five orbits in time-tag mode. The uncertainty of the transit midpoint time propagated to our latest exposure (21 August 2019) is $7.6 \mathrm{~min}$, based on the ephemeris of Bonomo et al. (2017). The COS observations were set to spectroscopic element G130M centered at $1291 \AA$ and a circular aperture with diameter 2.5 arcsec, yielding wavelength ranges [1134, 1274] $\AA$ and [1290, 1429] $\AA$. The STIS observations were set to spectroscopic element G140M centered at 1222 
Table 2. Observations log of WASP-29 in the HST PanCET program.

\begin{tabular}{|c|c|c|c|c|}
\hline Visit & Orbit & $\begin{array}{l}\text { Start time } \\
(\mathrm{UT})\end{array}$ & $\begin{array}{l}\text { Exp. time } \\
\text { (s) }\end{array}$ & $\begin{array}{c}\text { Phase } \\
\text { (h) }\end{array}$ \\
\hline \multicolumn{5}{|c|}{ COS spectroscopy } \\
\hline \multirow{5}{*}{ A } & 1 & $2018-05-19 \quad 15: 49: 32$ & 2182.144 & -3.54 \\
\hline & 2 & 2018-05-19 17:08:21 & 2706.112 & -2.01 \\
\hline & 3 & 2018-05-19 18:43:40 & 2706.112 & -0.42 \\
\hline & 4 & 2018-05-19 20:20:47 & 2706.176 & +1.20 \\
\hline & 5 & 2018-05-19 22:01:53 & 2706.176 & +2.85 \\
\hline \multirow{5}{*}{ B } & 1 & $2018-07-17$ 12:30:51 & 2182.112 & -3.97 \\
\hline & 2 & 2018-07-17 13:56:29 & 2706.144 & -2.47 \\
\hline & 3 & $2018-07-17$ 15:31:55 & 2706.176 & -0.88 \\
\hline & 4 & 2018-07-17 17:09:16 & 2706.112 & +0.74 \\
\hline & 5 & 2018-07-17 18:48:57 & 2706.144 & +2.40 \\
\hline \multirow{5}{*}{$\mathrm{C}$} & 1 & 2018-11-04 09:01:12 & 2182.208 & -4.48 \\
\hline & 2 & 2018-11-04 10:29:04 & 2706.208 & -3.09 \\
\hline & 3 & 2018-11-04 12:04:32 & 2706.208 & -1.51 \\
\hline & 4 & 2018-11-04 13:41:15 & 2706.176 & +0.11 \\
\hline & 5 & 2018-11-04 15:20:13 & 2706.208 & +1.80 \\
\hline \multicolumn{5}{|c|}{ STIS spectroscopy } \\
\hline \multirow{5}{*}{$\mathrm{D}$} & 1 & 2019-08-09 20:50:56 & 1751.159 & -3.90 \\
\hline & 2 & 2019-08-09 22:14:23 & 1983.173 & -2.48 \\
\hline & 3 & 2019-08-09 23:49:48 & 1983.189 & -0.89 \\
\hline & 4 & 2019-08-10 01:25:12 & 1983.179 & +0.70 \\
\hline & 5 & 2019-08-10 03:00:36 & 1983.195 & +2.29 \\
\hline \multirow{5}{*}{$\mathrm{E}$} & 1 & $2019-08-17$ 17:54:04 & 1751.138 & -3.14 \\
\hline & 2 & 2019-08-17 19:21:08 & 1983.132 & -1.66 \\
\hline & 3 & 2019-08-17 20:56:28 & 1983.115 & -0.07 \\
\hline & 4 & 2019-08-17 22:31:47 & 1983.187 & +1.52 \\
\hline & 5 & 2019-08-18 00:07:06 & 1983.183 & +3.11 \\
\hline \multirow{5}{*}{$\mathrm{F}$} & 1 & $2019-08-21$ 15:37:18 & 1751.147 & -3.57 \\
\hline & 2 & 2019-08-21 17:04:49 & 1983.181 & -2.08 \\
\hline & 3 & 2019-08-21 18:40:08 & 1983.015 & -0.49 \\
\hline & 4 & $2019-08-2120: 15: 27$ & 1983.193 & +1.10 \\
\hline & 5 & 2019-08-21 21:50:46 & 1983.197 & +2.69 \\
\hline
\end{tabular}

Notes. Phases are in relation to the orbit of WASP-29 b.

$\AA$ and a slit with dimensions $52 \times 0.1$ arcsec (the observations log is shown in Table 2), yielding a wavelength range [1194, 1248] A.

\subsection{Data reduction}

The COS data were reduced automatically by the instrument pipeline. The STIS data required some custom reduction due to strong background contamination in some exposures. The custom STIS reduction consists in using the pipeline flat-fielded frames to extract the spectra and perform background removal. We extracted the spectra following a similar procedure as in dos Santos et al. (2020b), in which we manually set the crossdispersion positions of the spectra and the background; the extraction box width was kept the same as in the pipeline reduction. We determined, by visual inspection, the spectrum crossdispersion positions to be $y=189 \mathrm{px}$ and set the background to be centered $20 \mathrm{px}$ away from the spectrum. We combined all STIS exposures to produce a high signal-to-noise Lyman- $\alpha$ spectrum, which is shown in Fig. 2 We used the time-tag information of the STIS and COS exposures to produce four subexposures per orbit.

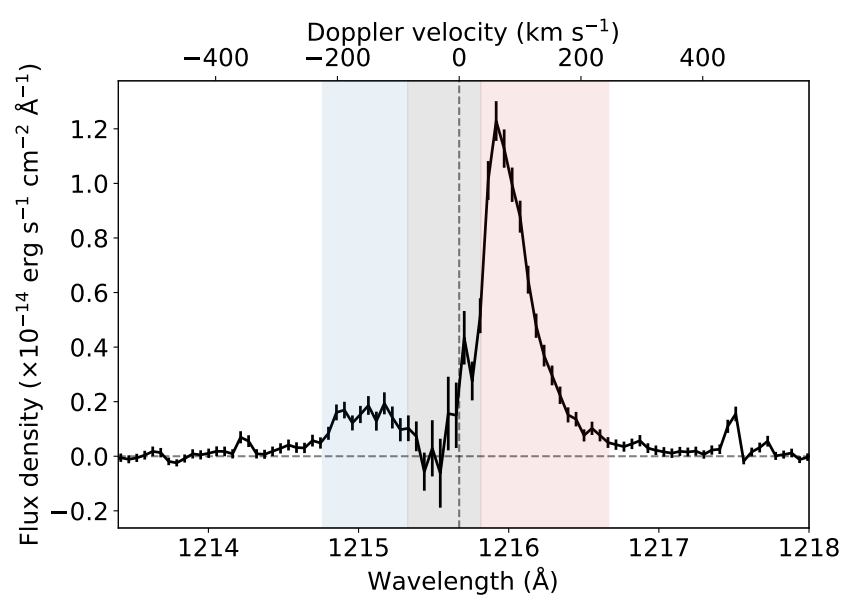

Fig. 2. Observed Lyman- $\alpha$ spectrum of WASP-29 with HST-STIS in a total exposure time of 8 hours. The gray band represents the wavelength span with strong geocoronal contamination (removed in data reduction). The blue and red regions represent the respective blue and red wings. The Doppler velocities are in the stellar rest frame.

\subsection{Treatment of uncertainties}

WASP-29 is a faint star in FUV, which means the observed count rates are limited to just a few photons per exposure (except for the Lyman- $\alpha$ line, which is the brightest feature in this wavelength region). In this regime, the uncertainties of the flux measurements do not follow a Gaussian distribution, but rather a Poisson distribution. Both pipelines of STIS and COS (respectively calstis and calcos) can calculate the uncertainties of FUV observations using Poisson confidence intervals. In particular, calcos produces confidence intervals calculated with the methodology outlined in Gehrels (1986), while calstis returns confidence intervals assuming Gaussian noise 1 .

Due to the non-Gaussian nature of these uncertainties, care has to be taken when propagating them. This is particularly important when calculating the final uncertainties of, for example, transmission spectra, fluxes inside a pass band that is several pixels wide, or combined exposures to produce a higher signal-tonoise spectrum. It is unclear if this is the same effect seen by Wilson et al. (2017), who reported overestimated flux uncertainties produced by the calcos pipeline.

In this work, when computing light curves of spectral lines and combining spectra from COS, we work with the raw or net counts and, at the final step, convert them to the physical flux in order to perform a proper treatment of uncertainties. The $1 \sigma$ uncertainties are calculated from the confidence intervals of a Poisson distribution based on the raw number of counts registered in the detector, which includes background sources. The conversion between counts and physical flux is performed using the tabulated sensitivity of the detector. The STIS Lyman- $\alpha$ spectra possess count rates at least one order of magnitude higher than those of COS, so the assumption of Gaussian uncertainties is appropriate for STIS spectra.

\footnotetext{
${ }^{1}$ Except for time-tag split data processed with the inttag method of calstis, which also produces uncertainties based on the methodology of Gehrels (1986).
} 


\subsection{Search for instrumental systematics}

The engineering jitter files of HST contain telemetry and pointing information that can be used to understand potential systematic trends in long-slit observations with STIS. This method was first used by Sing et al. (2019) and we apply our implementation of the technique. The main source of systematic trends in STIS data is slit losses, which are caused by two effects: i) changes in the position of the target star in the slit, both in the dispersion and cross-dispersion directions, and ii) changes in the point-spread function (PSF) seen by the detector due to thermal variations of the telescope - also known as breathing. Previous ultraviolet studies with HST have shown that these systematic trends are more important the brighter is the observed star (e.g., Bourrier et al. 2017c, 2018a; Sing et al.2019), and are not present in COS spectroscopy (dos Santos et al. 2019).

The pointing of the telescope can be traced by the right ascension and declination data present in the jitter data, but what matters in our case is the position of the target in relation to the slit. While we do not know exactly where our target is in relation to the slit at a sub-pixel level, we can gather this information from the jitter data of the guide stars. Usually, HST observations are performed with two guide stars, called dominant and roll; their positions in relation to the $\mathrm{V}_{2}$ and $\mathrm{V}_{3}$ axes of the telescope are traced to sub-pixel levels; since the slit is not aligned to the reference axes of the telescope, we need to rotate the dominant and roll position vectors to the slit reference frame - we call these new vectors $\mathrm{V}_{\mathrm{d}}$ (dispersion direction) and $\mathrm{V}_{\mathrm{s}}$ (spatial, or cross-dispersion direction).

In order to infer if the jitter trends cause slit losses in the STIS exposures, we searched for correlations between the observed Lyman- $\alpha$ red wing emission fluxes of the time-tag split data and the jitter data using the Spearman- $r$ coefficient (Spearman 1904). The results are shown in Table 3. Despite Visit E displaying the strongest jitter trends, the correlation with the Lyman- $\alpha$ fluxes are weak, with $p$-values above 0.10 . In relative terms, the strongest (anti-)correlations are seen in Visit D, where the guide star $\mathrm{V}_{\mathrm{d}}$ vector and the declination have Spearman- $r$ coefficients around 0.50 in modulus. Nevertheless, in absolute terms, these coefficients are below the threshold of 0.75 in modulus to be considered significant. Thus we conclude that there are negligible jitter trends in our STIS dataset.

We also searched for breathing effects by phase folding the subexposures to the orbit of HST and comparing the data to Fourier decomposition models (as in Bourrier et al. 2017c). We evaluated the likelihood of breathing effects by comparing the Bayesian Information Criterion (BIC) of models with varying orders fitted to the observed data. In all visits a flat model with no systematics is favored against higher order models: $\triangle \mathrm{BIC}=20$ for Visit $\mathrm{D}, \Delta \mathrm{BIC}=9$ for Visit $\mathrm{E}$, and $\triangle \mathrm{BIC}=12$ for Visit $\mathrm{F}$.

\section{Lyman- $\alpha$ emission time series}

The Lyman- $\alpha$ emission line $\left(\mathrm{HI}_{\mathrm{I}}\right)$ is the strongest feature in the far-ultraviolet spectrum of cool stars (Linsky|2017) and plays an important role in the photochemistry of planetary atmospheres (Segura et al. 2005). For stars other than the Sun, the Lyman- $\alpha$ emission is partially or completely absorbed by the interstellar medium (ISM). For the nearest stars, the core of the line is inaccessible, but its wings can still be observed, yielding the usual double-peaked profile (Fig. 2). The shape of this observed profile will depend on the properties of the intrinsic stellar emission, the physical properties of the ISM, and the radial velocity of the observed star.
Atmospheric signatures in hot transiting exoplanets have been previously detected as a large $(>10 \%)$ absorption in the Lyman- $\alpha$ line, which traces exospheric H I atoms (e.g., VidalMadjar et al. 2003, Lecavelier des Etangs et al. 2012; Ehrenreich et al. 2015; Bourrier et al. 2018b). Such a signal indicates the presence of a H-rich exospheric cloud being accelerated by radiation pressure and interactions with the stellar wind through charge exchange. We searched for a similar feature in the Lyman- $\alpha$ transit time series of WASP-29. At first, this search was performed by visual inspection of the Lyman- $\alpha$ profiles (Fig. 3. Upon concluding that there was no obvious signal, we performed a light curve analysis of the fluxes in the blue and red wing bandpasses. We define these bandpasses by the limits of the geocoronal contamination $\left([-60,+60] \mathrm{km} \mathrm{s}^{-1}\right.$ in the heliocentric restframe, or $[-84,+35] \mathrm{km} \mathrm{s}^{-1}$ in the stellar restframe) and the approximate limits where the stellar flux is above the $1 \sigma$ noise floor of the instrument. This yields the blue and red wing bandpass limits as $[-224,-84] \mathrm{km} \mathrm{s}^{-1}$ and $[+35,+245] \mathrm{km} \mathrm{s}^{-1}$, respectively.

In a single STIS exposure, we can measure the Lyman- $\alpha$ blue wing flux with a mean precision of $41.6 \%, 30.4 \%$, and $29.1 \%$, respectively for visits $\mathrm{D}, \mathrm{E}$, and $\mathrm{F}$. In the case of the red wing fluxes, the mean precision values are $10.4 \%, 9.5 \%$, and $9.9 \%$, respectively. Due to potential stellar flux modulation in the time scales that separate the visits, we normalize each time series by the out-of-transit flux. This baseline is measured as the average flux in orbits 1,2 , and 5 of each visit. We fit the normalized light curves to transit models calculated with batman (Kreidberg 2015) and estimate the uncertainties of the fit using the Markov chain Monte Carlo ensemble sampler implementation of emcee (Foreman-Mackey et al. 2013). We fix the orbital parameters of WASP-29 $\mathrm{b}$ to the values shown in Table 1 and vary only the depth $\left(R_{\mathrm{p}} / R_{*}\right)^{2}$.

There is a flux decrease of $\sim 20 \%$ in the Lyman- $\alpha$ red wing in Visit D, a feature that is seen in the in- and out-of-transit Lyman$\alpha$ profiles in Fig. 3, and the light curve of the red wing in Fig. 4 For comparison, GJ 436 shows an episodic red wing in-transit absorption as well (dos Santos et al.2019), but its origin remains unknown. Since this feature is not repeated in the other STIS observations of our program, we deem it unlikely to arise from the exosphere of WASP-29 b. Since the absorption takes place near the core of the Lyman- $\alpha$ emission of WASP-29, it is possible that the decrease in flux can be caused by stellar activity modulation. Similar to the case of HD 97658 in Bourrier et al. (2017c), stellar activity is expected to modulate the wavelength region nearest to the line core, which is formed at higher temperatures in the stellar corona, than its wings.

We did not find evidence to support Lyman- $\alpha$ flux absorption during the transit of WASP-29 b. Using the technique described above and by combining all the visits, we rule out any in-transit absorption depth larger than $49 \%(92 \%)$ in the blue wing at $1 \sigma$ $(3 \sigma)$ confidence. For the red wing light curves, we rule out any in-transit absorption signals above $9 \%(19 \%)$ at $1 \sigma(3 \sigma)$ confidence.

For comparison, the $\mathrm{H}_{\mathrm{I}}$ detection in HD $209458 \mathrm{~b}$ showed an in-transit depth of $15 \% \pm 4 \%$ in two bandpasses near the line core, between Doppler velocities $-130 \mathrm{~km} \mathrm{~s}^{-1}$ and $+100 \mathrm{~km}$ $\mathrm{s}^{-1}$ (excluding the wavelength interval with geocoronal emission contamination; Vidal-Madjar et al. 2003). The hot-Jupiter HD 189733 b displays $H_{\text {I }}$ transit depths of $14.4 \% \pm 3.6 \%$ between Doppler velocities $-230 \mathrm{~km} \mathrm{~s}^{-1}$ and $-140 \mathrm{~km} \mathrm{~s}^{-1}$ (Bourrier et al. 2013). The warm Neptunes GJ $436 \mathrm{~b}$ and GJ $3470 \mathrm{~b}$ display Lyman- $\alpha$ transit depths around 50\% in the Doppler velocity in- 
Table 3. Correlation between Lyman- $\alpha$ red wing fluxes and jitter data.

\begin{tabular}{l|cc|cc|cc}
\hline \hline & \multicolumn{2}{|c|}{ Visit D } & \multicolumn{2}{c|}{ Visit E } & \multicolumn{2}{c}{ Visit $\mathrm{F}$} \\
& Spearman- $r$ & $p$-value & Spearman- $r$ & $p$-value & Spearman- $r$ & $p$-value \\
\hline $\mathrm{V}_{\mathrm{d}, \text { dom }}$ & -0.547 & 0.012 & +0.223 & 0.346 & -0.293 & 0.210 \\
$\mathrm{~V}_{\mathrm{d}, \text { roll }}$ & -0.505 & 0.023 & +0.206 & 0.384 & -0.200 & 0.398 \\
$\mathrm{~V}_{\mathrm{s}, \text { dom }}$ & -0.030 & 0.900 & -0.260 & 0.269 & -0.186 & 0.431 \\
$\mathrm{~V}_{\mathrm{s}, \text { roll }}$ & +0.164 & 0.490 & +0.268 & 0.254 & +0.006 & 0.980 \\
Longitude & -0.090 & 0.705 & +0.147 & 0.535 & +0.268 & 0.254 \\
Latitude & -0.017 & 0.945 & -0.376 & 0.102 & -0.134 & 0.574 \\
Right ascension & +0.365 & 0.113 & -0.129 & 0.587 & +0.487 & 0.029 \\
Declination & -0.564 & 0.010 & +0.202 & 0.394 & -0.215 & 0.363 \\
\hline
\end{tabular}
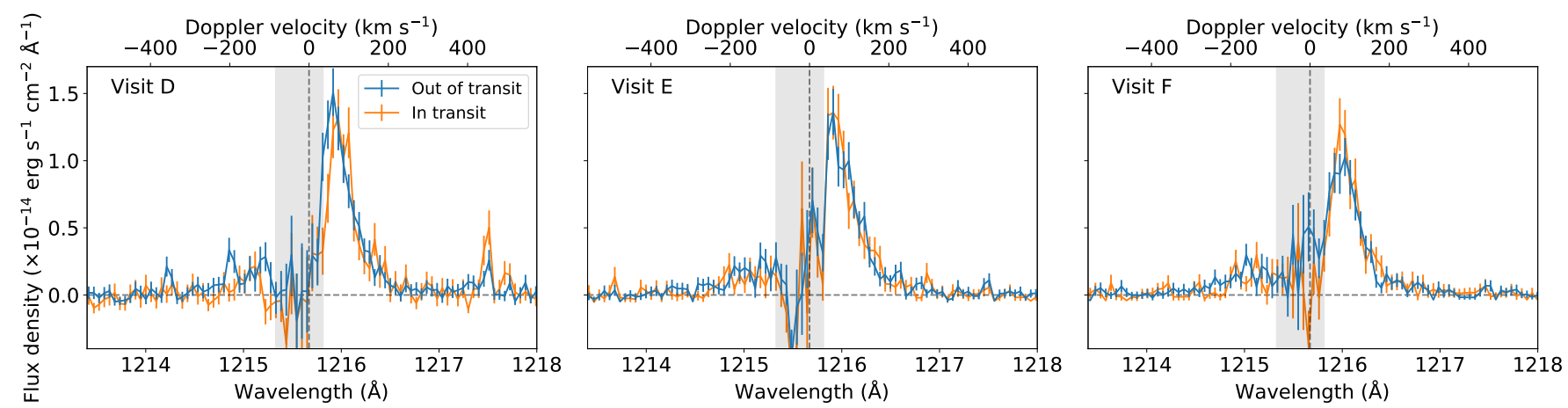

Fig. 3. In- and out-of-transit Lyman- $\alpha$ spectra of WASP-29 in Visits D, E, and F. The shaded region represents the interval where there is strong geocoronal contamination. We do not detect an obvious in-transit absorption signal.

terval [-120, -40] $\mathrm{km} \mathrm{s}^{-1}$ (Ehrenreich et al. 2015; Bourrier et al. 2018b).

\section{Metallic species time series}

To date, all detections of exospheric metals in transiting planets have been found in the hot Jupiters HD 209458 b (VidalMadjar et al.2004, Linsky et al. 2010, Vidal-Madjar et al.2013), HD 189733 b (Ben-Jaffel \& Ballester 2013), WASP-12 b (Fossati et al. 2010) and WASP-121 b (Sing et al. 2019). Sing et al. argue that exospheric metals in WASP-121 b can be observed in transmission when the exobase extends beyond the Roche lobe, allowing heavier species in the upper atmosphere to escape the planet. A search for such features in GJ $436 \mathrm{~b}$, which benefited from a more extensive dataset covering several years, resulted in non-detections only (Lavie et al. 2017; , Loyd et al. 2017; dos Santos et al. 2019). We perfomed a similar analysis for the three transits of WASP-29 b; the host star is fainter than GJ 436, thus we could analyze fewer spectral lines in the COS spectrum. The COS profiles of $\mathrm{C}_{\text {II, }} \mathrm{Si}$ III, Si IV, and $\mathrm{N} v$ are shown in Fig. 5, and their respective transit light curves are shown in Fig. 6

Similarly to the Lyman- $\alpha$ analysis, we fit the observed time series with transit light curve models to assess the absorption depth and its statistical significance. We found that the stellar flux in the line that arises from the ground-level state $\mathrm{C}_{\text {II }}$ (1334.5 $\AA$ ) decreases by $39 \%_{-11 \%}^{+12 \%}$; but we cannot rule out possible stellar variability effects that could mimic planetary signals since a similar "dip" is also seen before the transit between -5 and -4 hours in relation to the transit center. Furthermore, no clear-cut signal is seen in the combined $\mathrm{C}$ II spectra (Fig. 5). For comparison, Linsky et al. (2010) detected an in-transit absorption of $7.8 \% \pm 1.3 \%$ in the combined flux of the $\mathrm{C}_{\text {II }}$ doublet for HD 209458 b using COS spectra; that result matched well the marginal $2 \sigma$ detection of Vidal-Madjar et al. (2004), which was performed with the STIS spectrograph. García Muñoz et al. (2021) reported a $3.4 \sigma$ detection of excited-level $\mathrm{C}$ II during one transit of the super-Earth $\pi$ Men $\mathrm{c}$ that is consistent with ions filling the Roche lobe of the planet and moving away from the star. The decrease in C II flux that we observe in WASP-29 b is dominated by Visit A only, while Visits B and C are consistent with flat light curves. Since the signal in Visit A is not repeatable, we deem this decrease in flux as unrelated to the planet WASP-29 b.

The normalized light curve of the line arising from the excited-level $\mathrm{C}_{\text {II }}$ atom $(1335.7 \AA)$ is suggestive of a W-shaped time series. Such a transit light curve could be indicative of FUV limb-brightening in the stellar host, a well known phenomenon in the Sun (Minnis 1955). The effect is clearer when we look at the time-tag subexposures, which are shown as semi-transparent points in Fig. 6. This behavior would not arise from the shape of the planetary atmosphere, but from the limb-brightened chromosphere and transition region of the host star. However, more observations would be needed to confirm this feature for WASP$29 \mathrm{~b}$. Since the excited-level $\mathrm{C}_{\text {II }}$ line is the strongest feature in the COS spectra after Lyman- $\alpha$, it dominates the flux of the "all lines combined" light curve (lower right panel in Fig. 6) and the W-shape remains present. The COS light curves show that there are no obvious signatures of Si III, Si IV or $\mathrm{Nv}$ ions populating the exosphere of WASP-29 b. We calculated the $1 \sigma$ and $3 \sigma$ upper limits for the in-transit absorption depth and the observed COS fluxes, and the results are shown in Table 4

\section{The high-energy environment of WASP-29 b}

The STIS and COS observations provide useful information about the stellar UV irradiation and its impact on the planet upper atmosphere, as well as constraints on the ISM. They also al- 

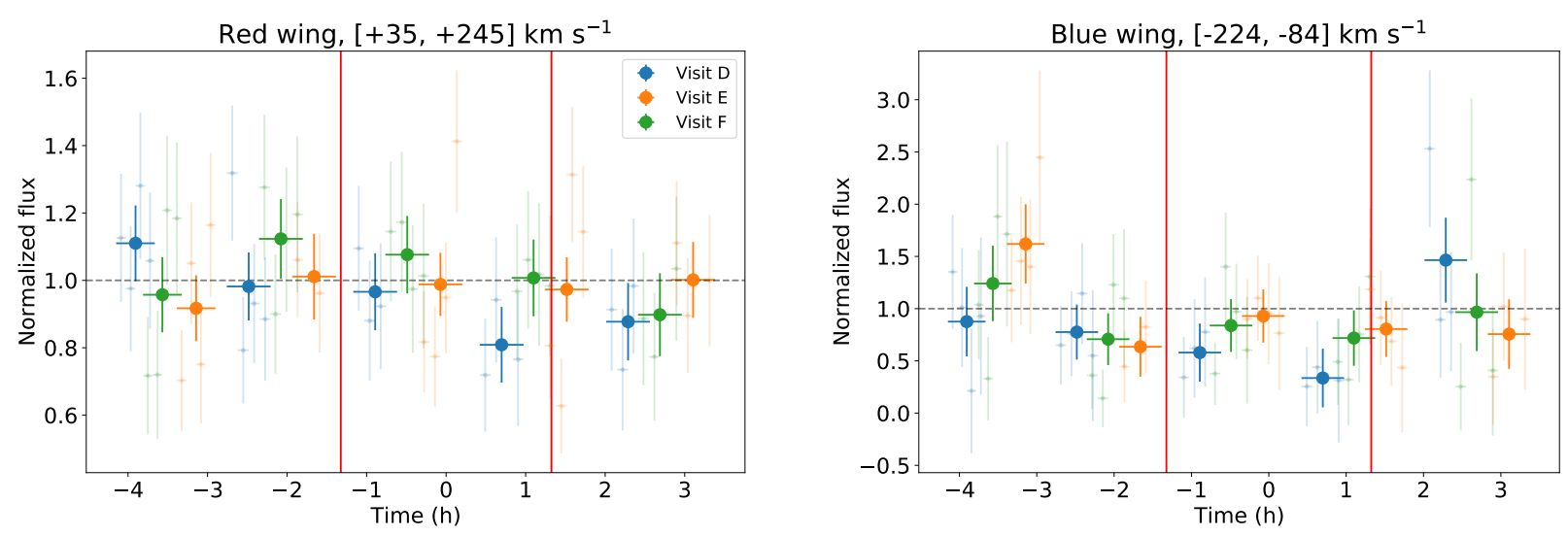

Fig. 4. Normalized Lyman- $\alpha$ light curves of WASP-29. The vertical red lines represent the times of first and fourth contacts of the transit of WASP-29 b. Each HST orbit is represented as a full symbol, while semi-transparent symbols represent sub-exposures within each orbit.
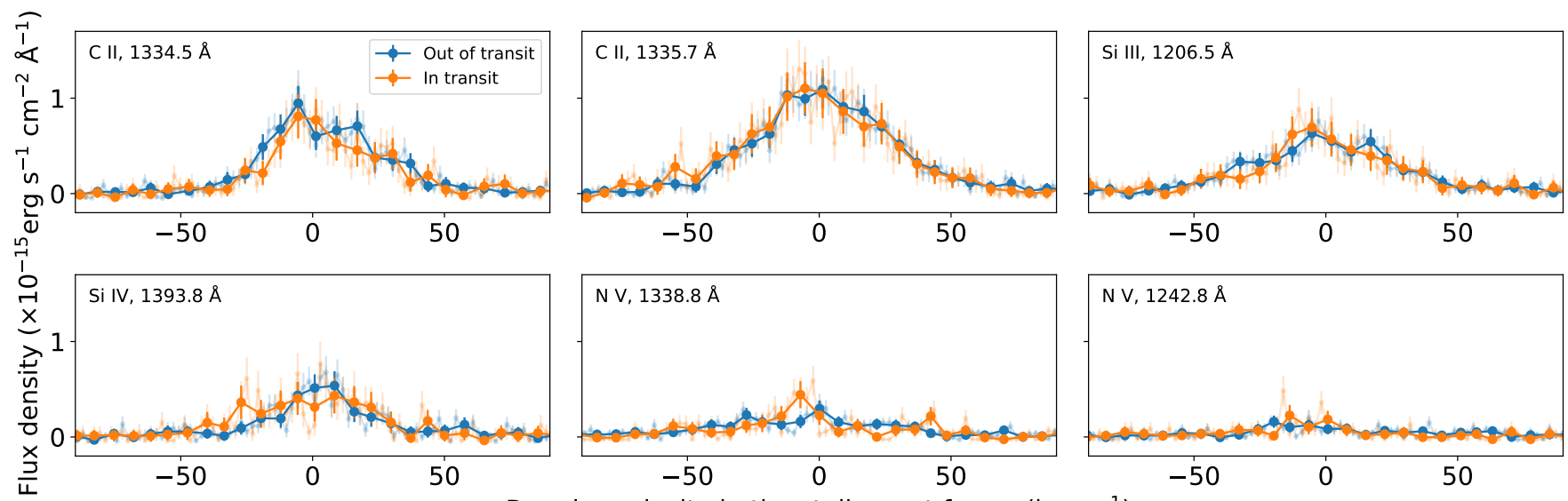

Fig. 5. In- and out-of-transit metallic spectral lines of WASP-29 in Visits A, B, and C.

Table 4. Observed fluxes of WASP-29 and upper limits of the in-transit absorption depth for WASP-29 b.

\begin{tabular}{lccccc}
\hline \hline Species & $\begin{array}{c}\text { Wavelength } \\
(\AA)\end{array}$ & $\begin{array}{c}\text { Integration range } \\
\left(\mathrm{km} \mathrm{s}^{-1}\right)\end{array}$ & $\begin{array}{c}\text { Flux } \\
\left(\times 10^{-16} \mathrm{erg} \mathrm{s}^{-1} \mathrm{~cm}^{-2}\right)\end{array}$ & \multicolumn{2}{c}{ Transit depth } \\
& 1334.5 & {$[-60,+60]$} & $1.94 \pm 0.11$ & $39 \%$ & $3 \sigma$ \\
\hline C $_{\text {II }}$ & 1335.7 & {$[-80,+80]$} & $2.88 \pm 0.12$ & $<10 \%$ & $39 \%{ }_{-31 \%}^{+40 \%}$ \\
C $_{\text {II }}$ & 1206.5 & {$[-80,+80]$} & $1.33 \pm 0.07$ & $11 \% \pm 9 \%$ & $<40 \%$ \\
Si III & 1393.8 & {$[-60,+60]$} & $0.92 \pm 0.07$ & $<17 \%$ & $<49 \%$ \\
Si i $_{\text {IV }}$ & 1238.8 & {$[-80,+80]$} & $0.63 \pm 0.04$ & $<31 \%$ & $<70 \%$ \\
$\mathrm{~N} \mathrm{~V}$ & & & & & $<$ \\
\hline
\end{tabular}

low us to interpret the non-detections of atmospheric signals and to predict signals that could be observed in other wavelengths. The ISM absorbs a large portion of the stellar emission at short wavelengths, including the Lyman- $\alpha$ emission and the extreme ultraviolet (EUV) spectrum, so they need to be reconstructed.

\subsection{Reconstruction of the intrinsic Lyman- $\alpha$ line}

We used the STIS observations of WASP-29 to reconstruct its intrinsic Lyman- $\alpha$ spectrum following the standard method used in, for example, Bourrier et al. (2017a) and Bourrier et al. (2018b). In short, we fit the observed spectrum to a model of the intrinsic emission line attenuated by ISM absorption ( $\mathrm{H}_{\mathrm{I}}$ and deuterium), scaled for distance, and convolved with the instrumental response. The model is oversampled in wavelength and rebinned over the STIS spectral table after convolution; the fit yields an estimate of the intrinsic emission and certain properties of the ISM in the line of sight.

Since no signatures of planetary absorption or stellar variability were found in any of the visits, the reconstruction was performed using all available spectra averaged into one master spectrum per visit. In all visits we excluded from the fit the velocity range $[-60,60] \mathrm{km} \mathrm{s}^{-1}$ (heliocentric rest frame), which is too contaminated by geocoronal emission. We fit the three master spectra together using the Markov chain Monte Carlo (MCMC) software package emcee (Foreman-Mackey et al. 2013). K dwarfs can show either double-peaked or single-peaked Lyman- $\alpha$ profiles, depending on the presence of a self-reversa ${ }^{2}$

2 A self-reversal in Lyman- $\alpha$ happens in some stars, like the Sun, because there is a layer in of absorbing material in the stellar atmosphere 

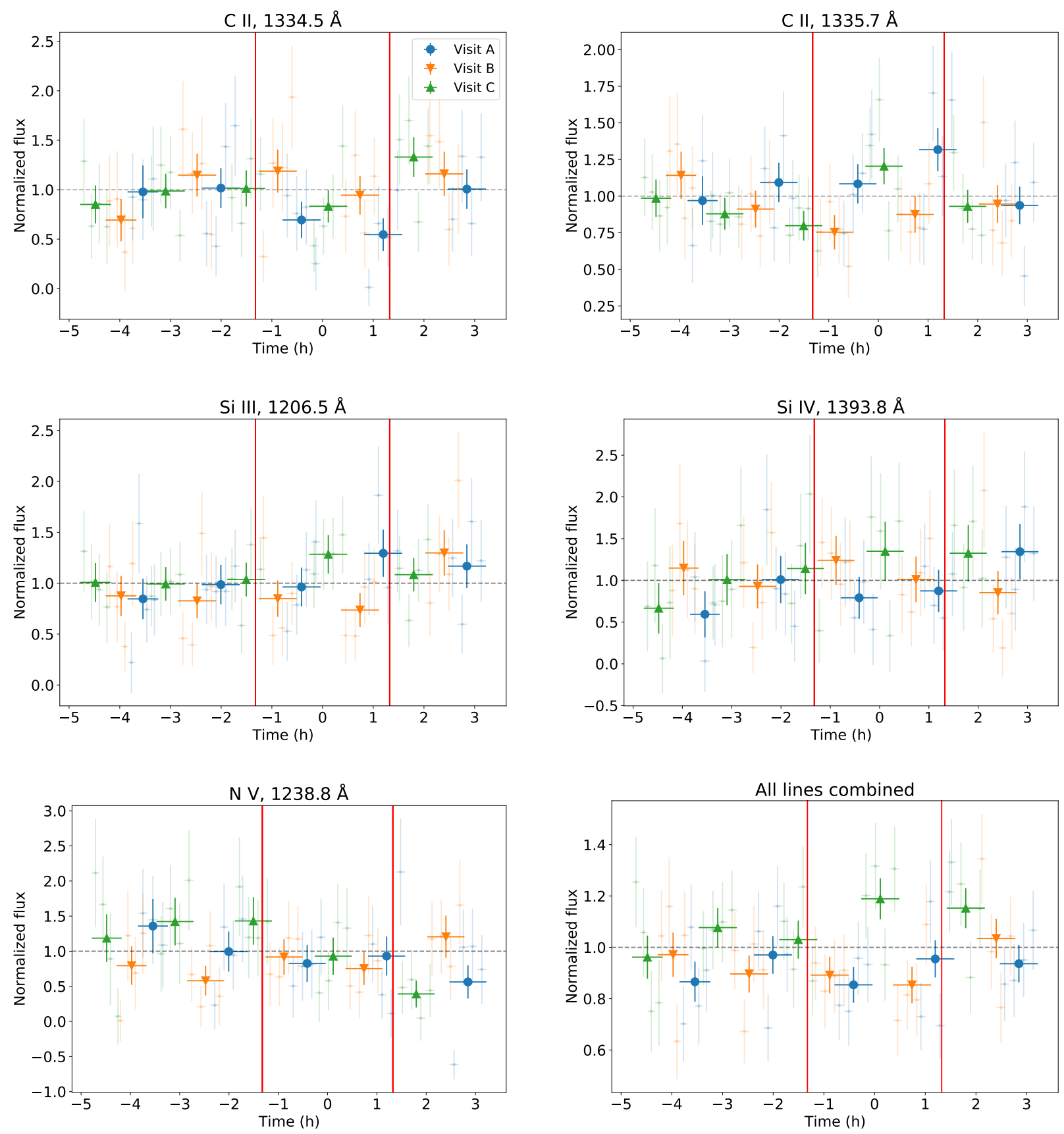

Fig. 6. Light curves of the metallic species emission lines of WASP-29 in the COS visits. The vertical red lines represent the times of first and fourth contacts of the transit of WASP-29 b. Each HST orbit is represented as a full symbol, while semi-transparent symbols represent sub-exposures within each orbit.

in their core (e.g., Wood et al. 2005, Youngblood et al. 2016 Bourrier et al. 2017b). We found that a single-peaked Voigt profile provides the best fit to the WASP-29 intrinsic Lyman- $\alpha$ line. The theoretical intrinsic line profile is defined by its centroid, its temperature (assuming pure thermal broadening), its Voigt damping parameter, and its total flux $F_{\mathrm{Ly}-\alpha}(1 \mathrm{au})$. Except for the total flux, all properties were set to the same value for the three visits. The line centroid was fixed to the systemic velocity of WASP-29 (24.48 $\mathrm{km} \mathrm{s}^{-1}$, as in Table 1). The absorption profile of the ISM along the line of sight is common to the three

between the region where the line is formed and the outermost stellar layers (see, e.g., Warren et al. 1998). visits, and defined by its column density of neutral hydrogen $\log _{10} N_{\mathrm{ISM}}(\mathrm{HI})$, its Doppler broadening parameter $b_{\mathrm{ISM}}(\mathrm{HI})$, and its heliocentric radial velocity $\gamma_{\mathrm{ISM} / \odot}$. The $\mathrm{DI} / \mathrm{H}_{\mathrm{I}}$ ratio was set to $1.5 \times 10^{-5}$ (e.g., Hébrard \& Moos 2003, Linsky et al. 2006). We adopted the Lyman- $\alpha$ oscillator strength $f=0.41641$ from the NIST database ${ }^{3}$. The Doppler broadening of the ISM toward WASP-29 is not constrained by its saturated Lyman- $\alpha$ line absorption profile, and was fixed to the value of the Local Interstellar Cloud (LIC).

3 Available in https://physics.nist.gov/PhysRefData/ASD/
lines_form.html 


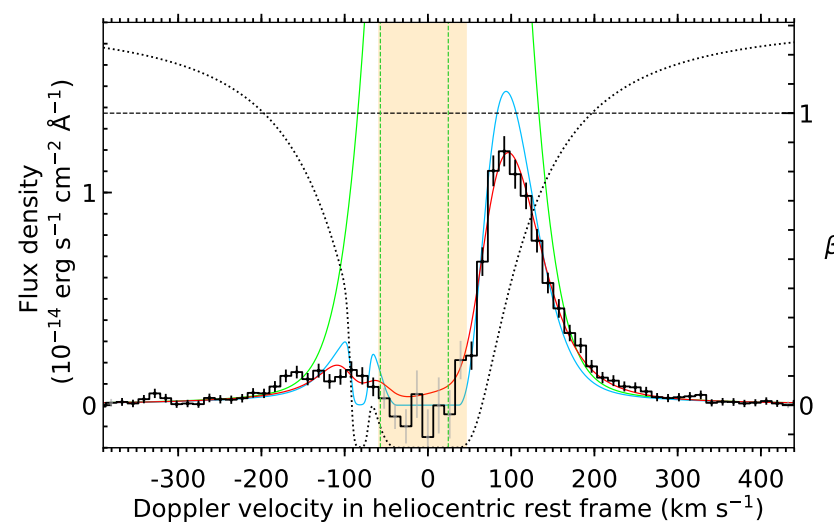

Fig. 7. Lyman- $\alpha$ line profile of WASP-29 averaged over the three visits. The black histogram shows the observed spectra, fit over points with black error bars. The orange band shows the region strongly contaminated by geocoronal emission to be excluded from the fit. The green full line is the best fit for the intrinsic stellar line profile, and the green dotted line represents the zero velocity in the stellar rest frame. It yields the blue profile after absorption by the interstellar medium, whose profile is plotted as a dotted black line (ISM absorption in the range 0-1 has been scaled to the vertical axis range). The red line shows the line profile fit to the data, after convolution with the STIS instrumental profile. The theoretical intrinsic stellar line profile also corresponds to the profile of the ratio $\beta$ between radiation pressure and stellar gravity, which is shown in the right $y$-axis.

We obtain a minimum $\chi^{2}$ of 166 for 151 degrees of freedom (reduced $\chi^{2}=1.1$ ). The best-fit model for the line averaged over the three visits is shown in Fig. 77. The LISM kinematic calculator ${ }^{4}$, a dynamical model of the local ISM (Redfield \& Linsky 2008), predicts that the line of sight toward WASP-29 crosses the LIC cloud with a heliocentric radial velocity of $-1.29 \mathrm{~km} \mathrm{~s}^{-1}$. This is in very good agreement with our derived value of $-0.1 \pm 2.6 \mathrm{~km} \mathrm{~s}^{-1}$. Our best-fit column density $\left(\log _{10} N_{\text {ISM }}\left(\mathrm{H}_{\mathrm{I}}\right)=18.55 \pm 0.04 \mathrm{~cm}^{-2}\right)$ is also consistent with the range of values expected for a star at a distance of $87.8 \mathrm{pc}$ (Fig. 14 in Wood et al.2005).

The total flux in the observed and reconstructed Lyman- $\alpha$ lines shows no significant variations between the three visits, strengthening the long-term stability of the stellar UV emission. The average line flux $\mathrm{F}_{\mathrm{Ly}-\alpha}(1 \mathrm{au})=17.6_{-1.6}^{+2.1} \mathrm{erg} \mathrm{s}^{-1} \mathrm{~cm}^{-2}$ yields a rotation period of about 20 days for WASP-29 from the empirical relation in Youngblood et al. (2016). In Fig. 7, the $\beta$ parameter is the ratio between radiation pressure and stellar gravity; our reconstruction shows that radiation pressure from WASP-29 Lyman- $\alpha$ line overcomes its gravity by a factor of seven at velocities between $[-100,100] \mathrm{km} \mathrm{s}^{-1}$ in the stellar rest frame. This is much larger than the factor 3 associated with the K0 dwarfs HD 189733 and HD 97658 (Bourrier et al.|2013, 2017c), or even the factor $\sim 4.5$ associated with the G0 dwarf HD 209458 (Bourrier \& Lecavelier des Etangs 2013), suggesting that the putative neutral hydrogen exosphere of WASP-29 b is quickly accelerated away from the planet in a strong regime of radiative blow-out.

\subsection{Reconstruction of the $X$-rays and extreme ultraviolet spectrum}

The results for COS observations allow us to provide constraints on the X-rays and extreme ultraviolet (X+EUV) emission of

\footnotetext{
4 http://sredfield.web.wesleyan.edu/
}

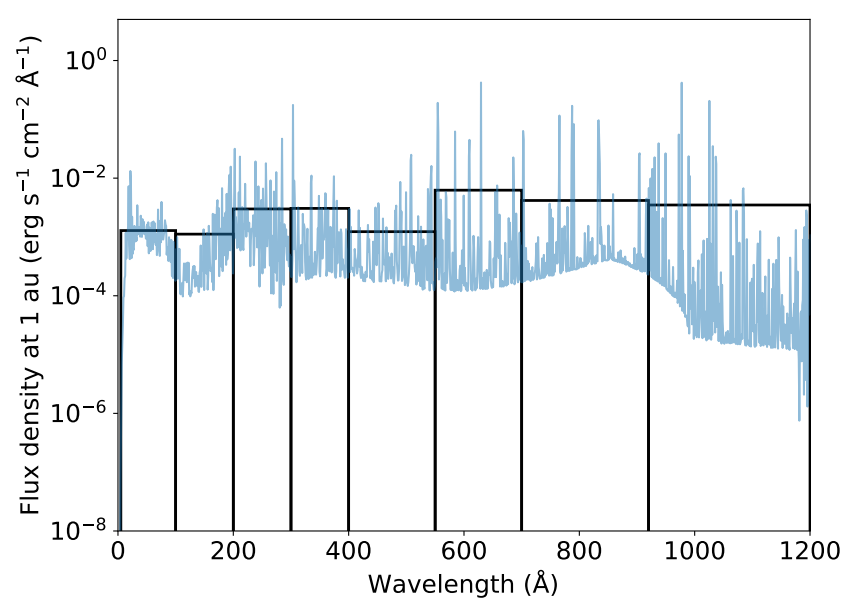

Fig. 8. Synthetic X+EUV spectrum of WASP-29 in high resolution (blue) and low resolution (black).

WASP-29, a part of its high-energy spectrum that is widely absorbed by the ISM and not straightforward to measure directly. WASP-29 was also observed by XMM-Newton (Prop. ID: 80479, PI J. Sanz-Forcada) in 14 May 2017. During the $6.9 \mathrm{ks}$ exposure only an upper-limit detection was made $(S / N=2)$ in the European Photon Imaging Camera (EPIC). We measured an X-ray luminosity of $L_{\mathrm{X}}<3.4 \times 10^{26} \mathrm{erg} \mathrm{s}^{-1}$ in the $0.12-$ $2.48 \mathrm{keV}$ spectral range (5-100 $)$. Based on this upper limit, we used a coronal model to construct the synthetic spectrum in the region 1-1200 , following Sanz-Forcada et al. (2011). We used the EPIC spectra to model the coronal temperatures, and the well-constrained COS fluxes (Sect. 4) to model the transition region. The model-derived fluxes at 1 au in different EUV bands are $2.74 \mathrm{erg} \mathrm{s}^{-1} \mathrm{~cm}^{-2}(100-920 \AA)$, and $0.78 \mathrm{erg} \mathrm{s}^{-1} \mathrm{~cm}^{-2}$ (100-504 $\AA$ ), for the first ionization edges of $\mathrm{H}$ and $\mathrm{He}$, respectively (Fig. 8). The resulting X+EUV (5-920 $⿱$ ) flux at 1 au is $3.98 \mathrm{erg} \mathrm{s}^{-1} \mathrm{~cm}^{-2}$. Further details of the coronal model will be given elsewhere (Sanz-Forcada et al., in prep.). The observed Xray emission is rather low for a K4V star (Pizzolato et al. 2003), which implies that WASP-29 is an aged star with a low state of activity.

Using the energy-limited approximation (Salz et al. 2016b), we estimate a total mass loss rate of WASP-29 b at $4 \times 10^{9} \mathrm{~g} \mathrm{~s}^{-1}$ for $15 \%$ escape efficiency. This value is intermediate when compared to the rates inferred from observations of the hot Jupiters HD 209458 b and HD 189733 b, and the warm Neptunes GJ $436 \mathrm{~b}$ and GJ $3470 \mathrm{~b}$, which range from $10^{8}$ to $10^{11} \mathrm{~g} \mathrm{~s}^{-1}$ (Lecavelier des Etangs et al. 2010, Bourrier \& Lecavelier des Etangs 2013; Bourrier et al. 2016, 2018b).

Similarly to Bourrier et al. (2017c), we estimate the photoionization rate and lifetime of atomic $\mathrm{H}$ around WASP-29 b based on the synthetic spectrum up to the ionization threshold of $911.8 \AA(13.6 \mathrm{eV})$. We found that the $\mathrm{H}_{\mathrm{I}}$ photoionization rate is $11.8 \times 10^{-5} \mathrm{~s}^{-1}$, yielding a photoionization lifetime of $2.35 \mathrm{~h}$. For comparison, the Hi lifetimes around the warm Neptunes with detected exospheres GJ $436 \mathrm{~b}$ and GJ $3470 \mathrm{~b}$ are $\sim 12 \mathrm{~h}$ and 55 minutes, respectively (Bourrier et al.2016, 2018b); in the case of the hot Jupiters with detected exospheres HD $209458 \mathrm{~b}$ and HD $189733 \mathrm{~b}$, the photoionization lifetimes are $\sim 8 \mathrm{~h}$ and $\sim 3.5 \mathrm{~h}$, respectively (Bourrier \& Lecavelier des Etangs 2013). The most likely explanation for the non-detection of exospheric $\mathrm{H} \mathrm{r}$ in WASP-29 b is a combination of the short photoionization 
lifetime, the fast acceleration of $\mathrm{H}$ atoms away from the planet by radiation pressure (also known as a "blow-out" regime), the low signal-to-noise ratio in the Lyman- $\alpha$ blue wing and the strong ISM absorption precluding access to velocities between [-84, $+35] \mathrm{km} \mathrm{s}^{-1}$. Thus, it is possible that WASP-29 b is, in fact, currently losing its atmosphere, but the high-energy environment around the planet and the distance of the system in relation to Earth are not conducive to produce detectable signals in FUV transmission spectroscopy.

One possible alternative to search for atmospheric escape signals in WASP-29 b is through the metastable He I triplet near $1.083 \mu \mathrm{m}$ (Seager \& Sasselov 2000, Oklopčić \& Hirata 2018 ) in transmission. This technique has been successfully applied in both space- and ground-based spectroscopy (e.g., Spake et al. 2018; Allart et al. 2018; Mansfield et al. 2018, Nortmann et al. 2018, Alonso-Floriano et al. 2019, Kirk et al. 2020). We computed the He I ionizing irradiation (up to 504 A) level of WASP-29 $\mathrm{b}$ using the synthetic spectrum, and found a value of $0.44 \mathrm{~W} \mathrm{~m}^{-2}$ at the planet's orbit $\left(440 \mathrm{erg} \mathrm{s}^{-1} \mathrm{~cm}^{-2}\right)$. For comparison, the hot Jupiter HD $209458 \mathrm{~b}$ receives an irradiation of approximately $1 \mathrm{~W} \mathrm{~m}^{-2}$, and its $\mathrm{He}_{\mathrm{I}}$ signal was challenging to measure (Alonso-Floriano et al. 2019); it is possible that a significant amount of observational effort may be required to measure He $\mathrm{I}$ in the extended atmosphere of WASP-29 b, since the intensity of the planetary signal in this line depends on the level of ionizing XUV irradiation received (Nortmann et al. 2018). The planets GJ 436 b, KELT-9 b (Nortmann et al.|2018), and WASP127 b (dos Santos et al. 2020a), which possess lower EUV irradiation levels than WASP-29 b, exhibit non-detections.

\section{Conclusions}

We observed six transits of WASP-29 b using the STIS and COS spectrographs installed on HST to search for signals of atmospheric escape in transmission. We did not find evidence for the presence of a Hi exosphere around WASP-29 b, likely due to a combination of factors: short Hi photoionization lifetime, a fast acceleration of $\mathrm{H}$ atoms away from the planet, and the low signal-to-noise ratio in the Lyman- $\alpha$ emission between Doppler velocities $[-100,+100] \mathrm{km} \mathrm{s}^{-1}$, which is the range where we expect such signal to appear.

An analysis of the stellar flux time series from COS spectra did not reveal significant, repeatable evidence for escaping metallic species in the atmosphere of WASP-29 b. The stellar line fluxes remain mostly stable over the three observing epochs. We found only a tentative in-transit absorption in the groundstate $C_{\text {II }}$ emission near $1334.5 \AA$, but we were unable to disentangle it from stellar activity effects. This result reinforces the requirement of repeatability for FUV atmospheric signals since this in-transit absorption is dominated by only one of the three visits. One way to assess if the observed signal comes from planetary absorption is to search for atmospheric escape using the $1.083 \mu \mathrm{m}$ metastable He triplet. If $\mathrm{He}$ is detected at high-altitudes around WASP-29 b, it is likely the planet is undergoing active atmospheric escape and thus would give support to the hypothesis of an atmospheric $\mathrm{C}_{\text {II }}$ signal.

Despite these non-detections, our observations allowed us to constrain the high-energy spectrum of WASP-29, as well as the environment around WASP-29 b. We reconstructed the intrinsic Lyman- $\alpha$ spectrum of WASP-29 using the STIS observations, and found that the Lyman- $\alpha$ flux is stable when we compare the three different epochs of observation spanning a baseline of 12 days. Furthermore, this results suggests that the level of Lyman- $\alpha$ radiation pressure efficiently overcomes gravity by a factor of seven, which is higher than those observed for other G- and K-type exoplanet hosts, such as HD 189733, HD 97658, and HD 209458. This puts the planet WASP-29 b in a regime of faster radiative acceleration of $\mathrm{H}$ atoms compared to other hot giant exoplanets for which we have observed signals of $\mathrm{H}$ i escape. Future observations of He I escape in the $1.083 \mu \mathrm{m}$ triplet may be able to provide better atmospheric escape constraints for WASP29 b. We also used XMM-Newton observations to constrain its EUV spectrum using a coronal model. The low levels of stellar activity in FUV, lack of stellar flares (in contrast to, for example, GJ 436), and the inferred low X-ray luminosity strongly suggest that WASP-29 is an old star. Based on the reconstructed EUV spectrum, we estimate that WASP-29 b is currently losing its atmosphere at a total rate of $4 \times 10^{9} \mathrm{~g} \mathrm{~s}^{-1}$, assuming a heating efficiency of $15 \%$. This mass loss rate is intermediate between the rates observed in hot Jupiters and warm Neptunes through Lyman- $\alpha$ spectroscopy.

Acknowledgements. L.d.S. thanks the valuable discussions with P. Wheatley on the results of this manuscript. G.W.H. acknowledges long-term support from NASA, NSF, Tennessee State University, and the State of Tennessee through its Centers of Excellence program. A.L.d.E. acknowledges the CNES for financial support. J.S.F. acknowledges support from the Spanish State Research Agency projects AYA2016-79425-C3-2-P and PID2019-109522GB-C51. We are grateful for the helpful feedback provided by the anonymous referee. This project has received funding from the European Research Council (ERC) under the European Union's Horizon 2020 research and innovation programme (project Four AcES grant agreement No 724427; project SPICE DuNE grant agreement No 947634), and it has been carried out in the frame of the National Centre for Competence in Research PlanetS supported by the Swiss National Science Foundation (SNSF). This research is based on observations made with the NASA/ESA Hubble Space Telescope. The data are openly available in the Mikulski Archive for Space Telescopes (MAST), which is maintained by the Space Telescope Science Institute (STScI). STScI is operated by the Association of Universities for Research in Astronomy, Inc. under NASA contract NAS 5-26555. This research made use of the NASA Exoplanet Archive, which is operated by the California Institute of Technology, under contract with the National Aeronautics and Space Administration under the Exoplanet Exploration Program. We used the open source software SciPy (Jones et al. 2001), Jupyter (Kluyver et al. 2016), Astropy (Astropy Collaboration et al. 2013), Matplotlib (Hunter 2007), batman (Kreidberg 2015), and emcee (Foreman-Mackey et al. 2013).

\section{References}

Allan, A. \& Vidotto, A. A. 2019, MNRAS, 490, 3760

Allart, R., Bourrier, V., Lovis, C., et al. 2018, Science, 362, 1384

Alonso-Floriano, F. J., Snellen, I. A. G., Czesla, S., et al. 2019, A\&A, 629, A110 Armstrong, D. J., Lopez, T. A., Adibekyan, V., et al. 2020, arXiv e-prints, arXiv:2003.10314

Astropy Collaboration, Robitaille, T. P., Tollerud, E. J., et al. 2013, A\&A, 558, A33

Beaugé, C. \& Nesvorný, D. 2013, ApJ, 763, 12

Ben-Jaffel, L. \& Ballester, G. E. 2013, A\&A, 553, A52

Bonomo, A. S., Desidera, S., Benatti, S., et al. 2017, A\&A, 602, A107

Bourrier, V., de Wit, J., Bolmont, E., et al. 2017a, AJ, 154, 121

Bourrier, V., Ehrenreich, D., Allart, R., et al. 2017b, A\&A, 602, A106

Bourrier, V., Ehrenreich, D., King, G., et al. 2017c, A\&A, 597, A26

Bourrier, V., Ehrenreich, D., Lecavelier des Etangs, A., et al. 2018a, A\&A, 615, A117

Bourrier, V. \& Lecavelier des Etangs, A. 2013, A\&A, 557, A124

Bourrier, V., Lecavelier des Etangs, A., Dupuy, H., et al. 2013, A\&A, 551, A63

Bourrier, V., Lecavelier des Etangs, A., Ehrenreich, D., et al. 2018b, A\&A, 620, A147

Bourrier, V., Lecavelier des Etangs, A., Ehrenreich, D., Tanaka, Y. A., \& Vidotto, A. A. 2016, A\&A, 591, A121

Cubillos, P., Erkaev, N. V., Juvan, I., et al. 2017, MNRAS, 466, 1868

dos Santos, L. A., Ehrenreich, D., Bourrier, V., et al. 2020a, A\&A, 640, A29 dos Santos, L. A., Ehrenreich, D., Bourrier, V., et al. 2020b, A\&A, 634, L4 dos Santos, L. A., Ehrenreich, D., Bourrier, V., et al. 2019, A\&A, 629, A47 Ehrenreich, D., Bourrier, V., Wheatley, P. J., et al. 2015, Nature, 522, 459 Ehrenreich, D. \& Désert, J.-M. 2011, A\&A, 529, A136

Ehrenreich, D., Lecavelier Des Etangs, A., Hébrard, G., et al. 2008, A\&A, 483, 933 
Foreman-Mackey, D., Hogg, D. W., Lang, D., \& Goodman, J. 2013, PASP, 125, 306

Fossati, L., Haswell, C. A., Froning, C. S., et al. 2010, ApJ, 714, L222

Gaia Collaboration, Brown, A. G. A., Vallenari, A., et al. 2018, A\&A, 616, A1

García Muñoz, A. 2007, Planet. Space Sci., 55, 1426

García Muñoz, A., Fossati, L., Youngblood, A., et al. 2021, arXiv e-prints, arXiv:2102.00203

García Muñoz, A. \& Schneider, P. C. 2019, ApJ, 884, L43

Gehrels, N. 1986, ApJ, 303, 336

Gibson, N. P., Aigrain, S., Barstow, J. K., et al. 2013, MNRAS, 428, 3680

Hébrard, G. \& Moos, H. W. 2003, ApJ, 599, 297

Helled, R., Lozovsky, M., \& Zucker, S. 2016, MNRAS, 455, L96

Hellier, C., Anderson, D. R., Collier Cameron, A., et al. 2010, ApJ, 723, L60

Hubbard, W. B., Hattori, M. F., Burrows, A., Hubeny, I., \& Sudarsky, D. 2007, Icarus, 187,358

Hunter, J. D. 2007, Computing In Science \& Engineering, 9, 90

Jin, S., Mordasini, C., Parmentier, V., et al. 2014, ApJ, 795, 65

Jones, E., Oliphant, T., Peterson, P., et al. 2001, SciPy: Open source scientific tools for Python

Kirk, J., Alam, M. K., López-Morales, M., \& Zeng, L. 2020, AJ, 159, 115

Kite, E. S., Fegley, Bruce, J., Schaefer, L., \& Ford, E. B. 2019, ApJ, 887, L33

Kluyver, T., Ragan-Kelley, B., Pérez, F., et al. 2016, in Positioning and Power

in Academic Publishing: Players, Agents and Agendas, ed. F. Loizides \& B. Schmidt, IOS Press, $87-90$

Kreidberg, L. 2015, PASP, 127, 1161

Kulow, J. R., France, K., Linsky, J., \& Loyd, R. O. P. 2014, ApJ, 786, 132

Kurokawa, H. \& Nakamoto, T. 2014, ApJ, 783, 54

Lavie, B., Ehrenreich, D., Bourrier, V., et al. 2017, A\&A, 605, L7

Lecavelier Des Etangs, A. 2007, A\&A, 461, 1185

Lecavelier des Etangs, A., Bourrier, V., Wheatley, P. J., et al. 2012, A\&A, 543, L4

Lecavelier des Etangs, A., Ehrenreich, D., Vidal-Madjar, A., et al. 2010, A\&A, 514, A72

Linsky, J. L. 2017, ARA\&A, 55, 159

Linsky, J. L., Draine, B. T., Moos, H. W., et al. 2006, ApJ, 647, 1106

Linsky, J. L., Yang, H., France, K., et al. 2010, ApJ, 717, 1291

Loyd, R. O. P., Koskinen, T. T., France, K., Schneider, C., \& Redfield, S. 2017, ApJ, 834, L17

Mansfield, M., Bean, J. L., Oklopčić, A., et al. 2018, ApJ, 868, L34

Matsakos, T. \& Königl, A. 2016, ApJ, 820, L8

Mazeh, T., Holczer, T., \& Faigler, S. 2016, A\&A, 589, A75

McDonald, G. D., Kreidberg, L., \& Lopez, E. 2019, ApJ, 876, 22

Minnis, C. M. 1955, Nature, 176, 652

Nortmann, L., Pallé, E., Salz, M., et al. 2018, Science, 362, 1388

Oklopčić, A. \& Hirata, C. M. 2018, ApJ, 855, L11

Owen, J. E. 2019, Annual Review of Earth and Planetary Sciences, 47, 67

Owen, J. E. \& Lai, D. 2018, MNRAS, 479, 5012

Pizzolato, N., Maggio, A., Micela, G., Sciortino, S., \& Ventura, P. 2003, A\&A, 397,147

Redfield, S. \& Linsky, J. L. 2008, ApJ, 673, 283

Rogers, L. A., Bodenheimer, P., Lissauer, J. J., \& Seager, S. 2011, ApJ, 738, 59

Salz, M., Czesla, S., Schneider, P. C., \& Schmitt, J. H. M. M. 2016a, A\&A, 586, A75

Salz, M., Schneider, P. C., Czesla, S., \& Schmitt, J. H. M. M. 2016b, A\&A, 585, L2

Sanz-Forcada, J., Micela, G., Ribas, I., et al. 2011, A\&A, 532, A6

Seager, S. \& Sasselov, D. D. 2000, ApJ, 537, 916

Segura, A., Kasting, J. F., Meadows, V., et al. 2005, Astrobiology, 5, 706

Sing, D. K., Lavvas, P., Ballester, G. E., et al. 2019, AJ, 158, 91

Spake, J. J., Sing, D. K., Evans, T. M., et al. 2018, Nature, 557, 68

Spearman, C. 1904, The American Journal of Psychology, 15, 72

Szabó, G. M. \& Kiss, L. L. 2011, ApJ, 727, L44

Tian, F. 2015, Annual Review of Earth and Planetary Sciences, 43, 459

Vidal-Madjar, A., Désert, J.-M., Lecavelier des Etangs, A., et al. 2004, ApJ, 604, L69

Vidal-Madjar, A., Huitson, C. M., Bourrier, V., et al. 2013, A\&A, 560, A54

Vidal-Madjar, A., Lecavelier des Etangs, A., Désert, J.-M., et al. 2003, Nature, 422,143

Vidotto, A. A. \& Cleary, A. 2020, arXiv e-prints, arXiv:2003.10272

Warren, H. P., Mariska, J. T., \& Wilhelm, K. 1998, ApJS, 119, 105

Wilson, P. A., Lecavelier des Etangs, A., Vidal-Madjar, A., et al. 2017, A\&A, 599, A75

Wood, B. E., Redfield, S., Linsky, J. L., Müller, H.-R., \& Zank, G. P. 2005, ApJS, 159,118

Youngblood, A., France, K., Loyd, R. O. P., et al. 2016, ApJ, 824, 101 